\title{
Changes in walking, body mass index, and cardiometabolic risk factors following residential relocation: Longitudinal results from the CARDIA study
}

\author{
Lindsay M. Braun ${ }^{\mathrm{a}, *}$, Daniel A. Rodriguez ${ }^{\mathrm{b}}$, Yan Song ${ }^{\mathrm{a}}$, Katie A. Meyer ${ }^{\mathrm{c}}$, Cora E. Lewis ${ }^{\mathrm{d}}$, \\ Jared P. Reis ${ }^{\mathrm{e}}$, Penny Gordon-Larsen ${ }^{\mathrm{c}, \mathrm{f}}$ \\ a Department of City and Regional Planning, University of North Carolina at Chapel Hill, United States \\ ${ }^{\mathrm{b}}$ Department of City and Regional Planning, University of California, Berkeley, United States \\ ${ }^{\mathrm{c}}$ Department of Nutrition, Gillings School of Global Public Health, University of North Carolina at Chapel Hill, United States \\ ${ }^{\mathrm{d}}$ Division of Preventive Medicine, Department of Medicine, University of Alabama at Birmingham, United States \\ e National Heart, Lung, and Blood Institute, Prevention and Population Sciences Program, United States \\ ${ }^{\mathrm{f}}$ Carolina Population Center, University of North Carolina at Chapel Hill, United States
}

\section{A R T I C L E I N F O}

\section{Available online 13 September 2016}

Keywords:

Built environment

Walkability

Walking

fixed effects

Residential self-selection

\begin{abstract}
A B S T R A C T
Background: While many studies have found the built environment to be associated with walking, most have used cross-sectional research designs and few have examined more distal cardiometabolic outcomes. This study contributes longitudinal evidence based on changes in walking, body mass index (BMI), and cardiometabolic risk following residential relocation.

Methods: We examined 1079 participants in the CARDIA study who moved residential locations between 2000 and 2006 (ages 32-46 in 2000, 49\% white/51\% black, 55\% female). We created a walkability index from measures of population density, street connectivity, and food and physical activity resources, measured at participants' pre- and post-move residential locations. Outcomes measured before and after the move included walking, BMI, waist circumference, blood pressure, insulin resistance, triglycerides, cholesterol, atherogenic dyslipidemia, and C-reactive protein. Fixed effects (FE) models were used to estimate associations between within-person change in walkability and within-person change in each outcome. These estimates were compared to those from random effects (RE) models to assess the implications of unmeasured confounding.

Results: In FE models, a one-SD increase in walkability was associated with a $0.81 \mathrm{mmHg}$ decrease in systolic blood pressure $[95 \% \mathrm{CI}:(-1.55,-0.07)]$ and a 7.36 percent increase in C-reactive protein $[95 \% \mathrm{CI}$ : $(0.60,14.57)]$. Although several significant associations were observed in the RE models, Hausman tests suggested that these estimates were biased for most outcomes. RE estimates were most commonly biased away from the null or in the opposite direction of effect as the FE estimates.

Conclusions: Greater walkability was associated with lower blood pressure and higher C-reactive protein in FE models, potentially reflecting competing health risks and benefits in dense, walkable environments. RE models tended to overstate or otherwise misrepresent the relationship between walkability and health. Approaches that base estimates on variation between individuals may be subject to bias from unmeasured confounding, such as residential self-selection.
\end{abstract}

(c) 2016 Elsevier Ltd. All rights reserved.

\section{Introduction}

The health benefits of physical activity have been well documented, with past research finding regular physical activity to be associated with lower risk of cardiovascular disease, type 2 diabetes, certain cancers, osteoarthritis, osteoporosis, and obesity, and with more favorable mental health outcomes (U.S. Department of Health and Human Services, 1996). Despite these widely acknowledged benefits,

\footnotetext{
* Correspondence to: Department of City and Regional Planning, University of North Carolina at Chapel Hill, Campus Box 3140, New East Building, Chapel Hill, NC 275993140, United States. Fax: +919 9625206.

E-mail address: maurerbraun@unc.edu (L.M. Braun).
} 
only one in five U.S. adults meets current physical activity guidelines (Centers for Disease Control and Prevention, 2015) and limited progress has been made during the past decade in increasing physical activity levels (Carlson et al., 2010). These observations make physical activity a critical focus for urban planning, engineering, and public health intervention.

As the field of public health has increasingly emphasized community-level interventions (Pikora et al., 2003) and the incorporation of physical activity into daily living (Sallis et al., 2006), interest has emerged in understanding how attributes of the built environment can facilitate active travel and thereby integrate physical activity into the context of daily routines. Research on the built environment and health has evolved from an early emphasis on access to recreational amenities to a more comprehensive consideration of land use and transportation characteristics (Harris et al., 2013). Recent research suggests that various measures of the built environment-including population density, land use mix, street connectivity, and composite measures of neighborhood walkability-are positively associated with walking (Van Dyck et al., 2010; Witten et al., 2012; Li et al., 2005) and overall physical activity (Van Dyck et al., 2010; Witten et al., 2012; Hoehner et al., 2011; Berke et al., 2007; Li et al., 2008; Garden and Jalaludin, 2008; King et al., 2005; Frank et al., 2004), and inversely associated with body mass index (BMI) (Hoehner et al., 2011; Berke et al., 2007; Li et al., 2008; Garden and Jalaludin, 2008; Frank et al., 2004; Joshu et al., 2008; Rundle et al., 2007). These findings suggest that the built environment may represent a promising intervention point for community-level efforts to promote physical activity and prevent obesity.

Additionally, epidemiological studies of the relationships of physical activity and body weight with cardiometabolic health outcomes (Green et al., 2014; Gordon-Larsen et al., 2009; Pires et al., 2012; Autenrieth et al., 2009) suggest that the built environment may also be associated with more distal measures of cardiometabolic risk. Relatively few studies have examined the relationship between the built environment and cardiometabolic risk factors (Leal and Chaix, 2011), although some research has found measures of neighborhood walkability to be favorably associated with blood pressure (Chaix et al., 2008; Agyemang et al., 2007), risk of type 2 diabetes (MüllerRiemenschneider et al., 2013), odds of metabolic syndrome (Baldock et al., 2012; Dengel et al., 2009), and other composite indicators of cardiometabolic risk (Coffee et al., 2013). At the same time, dense urban environments may entail cardiometabolic stressors such as noise, overcrowding, and air pollution (King, 2013), illustrating the potential competing health risks and benefits of walkable built environments.

Despite the fairly promising findings relating the built environment to health, this evidence base has relied largely on cross-sectional research designs that are vulnerable to a variety of shortcomings that limit the ability to make causal statements (Boone-Heinonen et al., 2011). For example, to the extent that individuals choose their residential neighborhoods based on their preferences or constraints related to walking and other health behaviors, the direction of causality between the built environment and health outcomes is ambiguous. Thus, corresponding estimates of the association between neighborhood walkability and health may be biased by residential self-selection.

Some methodological challenges may be more adequately addressed in longitudinal studies that evaluate changes in health following a change in the environment. "Fixed effects" and "random effects" models offer two different ways of analyzing longitudinal data. Fixed effects models examine within-person changes in exposures and outcomes over time, thereby controlling for characteristics-both measured and unmeasured-that remain constant (and whose effects remain constant) within individuals throughout the study period (Allison, 2005; Lovasi and Goldsmith, 2014). This method can be contrasted with random effects models, which examine differences both within and between individuals and therefore do not control for unmeasured characteristics that vary across individuals (Cameron and Trivedi, 2005). Fixed effects models may be useful in the presence of residential self-selection, as the determinants of neighborhood choice are difficult to measure and are thus generally unobserved.

Because changes to the built environment often take place slowly and incrementally, one alternative is to focus on individuals who move residential locations and thereby experience a distinct change in the built environment over relatively short periods of time. This approach does not fully resolve the endogeneity of residential choice and health, as individuals still choose whether and to which type of neighborhood to move. However, it does allow for the consideration of changes in exposures and outcomes within individuals over time. This research design has been used in several recent quasi-experimental studies that have measured neighborhood characteristics and health outcomes before and after residential relocation (Handy et al., 2008; Lee et al., 2009; Wells and Yang, 2008; Mumford et al., 2011; Giles-Corti et al., 2013; Christian et al., 2013; Hirsch et al., 2014). While the results have provided some evidence of longitudinal associations between the built environment and physical activity, walking, and BMI, these studies have often been limited by small samples of movers (Lee et al., 2009; Wells and Yang, 2008) or reliance on retrospective recall of behavior in the previous neighborhood environment (Handy et al., 2008; Mumford et al., 2011).

We build upon this emerging work using a sample of individuals who moved residential locations during six years of follow-up in the Coronary Artery Risk Development in Young Adults (CARDIA) study. Our objectives in this analysis were twofold. First, we used fixed effects models to evaluate whether within-person changes in walkability, brought about by residential relocation, were associated with within-person changes in walking physical activity, BMI, and several cardiometabolic risk factors. Specifically, we hypothesized that after conditioning on sociodemographic and health covariates, a move to a more walkable neighborhood would be positively associated with walking physical activity and high-density lipoprotein (HDL) cholesterol; and inversely associated with BMI, waist circumference, systolic blood pressure, insulin resistance, triglycerides, low-density lipoprotein (LDL) cholesterol, C-reactive protein (a marker of inflammation), and atherogenic dyslipidemia (a classification of cardiovascular disease risk based on elevated triglycerides and low HDL cholesterol). Second, we compared these estimates to those obtained from random effects models to examine potential differences in results when we did (fixed effects) versus did not (random effects) account for certain types of unmeasured confounding in our models. These comparisons contribute to an enhanced understanding of the relationship between the built environment, transportation, and health, and of the potential for bias from certain unobserved characteristics in this area of research.

\section{Data and methods}

\subsection{Study sample}

CARDIA is a population-based prospective epidemiologic study of the determinants and evolution of cardiovascular risk factors in young adults. In 1985-1986 (CARDIA exam year 0), 5115 eligible participants between the ages of 18 and 30 were recruited from the populations of Birmingham, Alabama; Chicago, Illinois; Minneapolis, Minnesota; and Oakland, California. Enrollment was designed to 
achieve balance by gender, race (black, white), education ( $\leq$ high school, $>$ high school), and age (18-24, 25-30) at each exam center. Specific recruitment procedures have been described previously (Hughes et al., 1987). Seven follow-up examinations have been conducted over 25 years. We used data from year 15 (2000-2001) and year 20 (2005-2006) to leverage a neighborhood environment questionnaire that was administered at the 2005-2006 exam. The retention rates for the two exam years were 74 percent and 72 percent of surviving cohort members, respectively.

The study sample was restricted to individuals who moved residential locations between the 2000-2001 exam (considered to be the "baseline" for this analysis) and the 2005-2006 exam (considered to be the "follow-up"). Among the 3169 respondents who participated in both exams, 1661 were excluded because they did not move and an additional 429 were excluded because they did not provide complete information on all variables of interest at both exam years. These exclusions resulted in a final analytic sample of 1079 cases, each observed at two time points.

To assess the potential implications of mover status and missing data, we compared our final analytic sample to (1) CARDIA participants who did not move residential locations and (2) CARDIA participants who moved but did not provide complete information on all variables of interest at both exam years. First, compared to non-movers, movers with complete data (i.e. the movers included in this sample) were younger, had lower income and educational attainment, and had smaller household sizes at baseline (Appendix Table A.1). A greater proportion of movers were male, black, unmarried, or current smokers; a greater proportion reported a health problem that interfered with physical activity; and a smaller proportion reported taking medication for cholesterol. The only statistically significant differences in baseline health between movers and non-movers were for triglycerides and atherogenic dyslipidemia, with movers having lower average triglycerides and a lower proportion of atherogenic dyslipidemia classification. Second, compared to movers excluded due to missing data, movers with complete data (i.e. the movers included in this sample) had slightly lower baseline BMI, waist circumference, systolic blood pressure, homeostasis model assessment of insulin resistance (HOMA-IR) (Matthews et al., 1985), and triglycerides, and slightly higher baseline HDL cholesterol and income (Appendix Table A.1). A greater proportion of included cases were employed at baseline, while a smaller proportion of included cases were classified as having atherogenic dyslipidemia or reported taking medication for cholesterol.

\subsection{Built environment exposure measure: walkability index}

The built environment exposure for this analysis was a composite walkability index created from measures of population density, street connectivity, and food and physical activity resources within three Euclidean (i.e. straight-line) kilometers of each respondent's residential location. We measured these attributes using time-varying Geographic Information System data linked to participants' geocoded home addresses at each exam.

Population density was calculated using Census data at the block group level. Street connectivity was measured as the number of street intersections divided by the three-kilometer area around each residential location (i.e. intersection density) and the ratio of street segments to intersections (i.e. link-to-node ratio), based on road network data from ESRI StreetMap. Although we could not collect finegrained land use data due to the geographic extent of the CARDIA sample, we had access to Dun \& Bradstreet (D\&B) (Dun \& Bradstreet, Inc., 2013) data describing the food and physical activity environment. D\&B is a commercial database of U.S. businesses and institutional facilities classified by their Standard Industrial Classification codes, and our subset of the data included businesses and facilities related to physical activity (e.g., parks, gyms, recreation centers, community centers, YMCAs) and the food environment (e.g., restaurants, grocery stores, supermarkets). We assumed that these physical activity and food resources would be correlated with the total amount of development in an area and created three specific measures to reflect the density and accessibility of resources. The first of these measures was a count of physical activity and food resources within three Euclidian kilometers of respondents' home addresses, which served as a proxy for the density of stores and activity amenities. To allow resources closer to home to contribute more to the count than those located farther away, resources within one kilometer were assigned a weight of one, while an inverse-distance weight was applied to resources beyond one kilometer. This weighting strategy is presented in Eq. (1):

Resource count $=\sum_{i=1}^{n} w_{i}$

where $i$ indexes individual physical activity and food resources and $w$ is a weight equal to 1 if distance $\leq 1 \mathrm{~km}$, or $\frac{1}{\text { distance( }(\mathrm{km})}$ if distance $>1 \mathrm{~km}$. To create the other two resource measures, we calculated the distance between a respondent's home address and every resource within three kilometers, and took the mean of these distance values as a measure of resource accessibility; this calculation was performed separately for physical activity and food resources. Collectively, the three resource measures allowed us to consider both the density of available resources and how proximate they were to CARDIA participants' residences.

The population density, street connectivity, and resource count measures were assumed to contribute positively to walkability. The mean distance measures were assumed to have a negative influence on walkability, as higher values implied that the average resource within the three-kilometer buffer was at a greater (i.e. less walkable) distance from a respondent's home. In calculating the composite index, we assigned weights to the individual components based on their relative importance in past research on the built environment and travel behavior. In a meta-analysis, Ewing and Cervero (2010) found the elasticity of walking to be greatest with respect to street connectivity, moderate for land use attributes, and lowest for measures of density. Thus, we assigned a weight of 3 to each of the street connectivity measures, a collective weight of 4 to the three food and physical activity resource measures (assumed to be correlated with development and land use), and a weight of 1 to the population density measure. Finally, since there were negative values, we added the absolute value of the sample minimum to each observation in order to place the walkability index on a scale starting at zero for the location(s) with the lowest walkability. The resulting index for each participant $i$ is presented in Eq. (2):

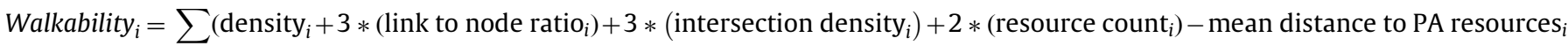

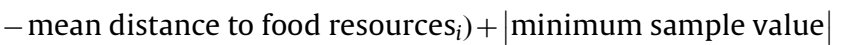

where the listed variables refer to standardized values across the full CARDIA sample. 
This walkability index for 2005-2006 was compared to an external measure, the 2013 Street Smart Walk Score ${ }^{\circledR}$, a widely used source for contemporary analyses of walkability; we could not use this external measure as the main exposure for our longitudinal study or for a comparative analysis of our 2000-2001 index because historical Street Smart Walk Score ${ }^{\circledR}$ data were not available. The traditional Walk Score ${ }^{\circledR}$ algorithm has been shown to be a valid indicator of neighborhood walkability (Duncan et al., 2011; Carr et al., 2010; Carr et al., 2011), and Street Smart Walk Score ${ }^{\circledR}$ offers a more complete measure of walkability by incorporating network rather than straight-line distances to amenities (Front Seat Management, 2014). We conducted this comparative analysis in a subset of CARDIA participants for whom 2013 Street Smart Walk Score ${ }^{\circledR}$ data were available $(n=1127)$. The walkability index in Eq. (2) was found to explain 66.4 percent of the variation in Street Smart Walk Score ${ }^{\circledR}$ using linear regression (data not shown). Furthermore, the walkability index and Street Smart Walk Score ${ }^{\circledR}$ were found to be comparable predictors of walking behavior as judged by model fit statistics (AIC, adjusted $\mathrm{R}^{2}$ ). We tested two additional weighting strategies for the index components: (1) no weights (i.e. all components weighted equally) and (2) street connectivity and resource measures weighted equally (both classes of measures weighted at 4, with population density still weighted at 1). All versions produced similar associations with Smart Walk Score ${ }^{\circledR}$ and walking behavior, and we retained the weights shown in Eq. (2) to be consistent with past research in this area (Ewing and Cervero, 2010). We also measured the walkability index within one kilometer of participants' home addresses, but the three-kilometer measure was a stronger predictor of both Street Smart Walk Score ${ }^{\circledR}$ and walking behavior. Thus, the three-kilometer walkability index was retained as the built environment exposure for this study.

\subsection{Health outcome measures: walking physical activity, BMI, and cardiometabolic risk factors}

At both exams, participants reported whether and how often they took walks or hikes or walked to work during the 12 months preceding the exam. We used this information to create two measures of walking-based physical activity. First, a dichotomous variable ("walking participation") indicated whether an individual reported engaging in any walking activity during the previous 12 months (1 if yes, 0 if no). Second, a continuous measure ("walking exercise units") accounted for the frequency and intensity of walking activity using the formula $4\left(m_{i}+3 n_{i}\right)$, where $m_{i}$ is the number of months of less frequent walking activity ( $<4$ hours/month) and $n_{i}$ is the number of months of more frequent walking activity ( $\geq 4$ hours/month) for individual $i$. The resulting values ranged from 0 to 144 units. This method has been used in past studies of the CARDIA cohort to offer an approximation of the metabolic equivalents (METs) derived from various types of physical activity (Jacobs et al., 1989).

BMI and waist circumference were calculated from anthropometry data recorded during each exam. Blood pressure was measured three times after a five-minute seated rest, and the average of the second and third readings was used. Continuous measures of insulin, glucose, triglycerides, LDL and HDL cholesterol, and C-reactive protein were derived from fasting blood samples. The insulin and glucose values were combined into a single measure of insulin resistance (HOMA-IR; lower values indicate better health); individuals who reported having doctor-diagnosed diabetes at baseline $(n=42)$ were removed from the HOMA-IR calculations and regressions, as HOMAIR calculations are inappropriate for those with diabetes. Based on the distribution of sample values, log transformations of HOMA-IR (baseline median $=1.75$, IQR $=1.37$ ), triglycerides (baseline median $=78, \mathrm{IQR}=57$ ), and C-reactive protein (baseline median $=1.37$, IQR $=3.13$ ) were used in the final regression models for this analysis. Atherogenic dyslipidemia was measured as a dichotomous variable indicating whether participants exhibited both of the following risk factors: elevated triglycerides ( $>150 \mathrm{mg} / \mathrm{dL}$ ) and low HDL cholesterol ( $<50 \mathrm{mg} / \mathrm{dL}$ for women, $<40 \mathrm{mg} / \mathrm{dL}$ for men).

\subsection{Covariates: sociodemographic characteristics, general health status, and reasons for moving}

We used information from interviewer-administered CARDIA questionnaires to measure sociodemographic characteristics (age, gender, race/ethnicity, educational attainment, income, household size, marital status, employment status), general health characteristics (smoking status, health problems interfering with physical activity), and reasons for moving to the current neighborhood. Since baseline age, gender, and race/ethnicity were constant over time, we treated them as fixed. We also specified educational attainment as timeinvariant because most participants had obtained their highest academic degree by the 2000-2001 exam. Race/ethnicity categories consisted of white and black, the only two categories included in the CARDIA cohort. Self-reported educational attainment (i.e. highest degree obtained) at the 2000-2001 exam was used to create a dichotomous indicator of whether an individual received a degree beyond high school.

Income, household size, marital status, employment status, smoking status, and general health status were time-varying characteristics that we included at each time point. Respondents reported their combined family income as falling into one of nine categories, and a measure in U.S. dollars was created as the midpoint of the selected income category. Household size was defined as the number of individuals living in the household. Participants were classified as married if they reported being currently married or living with someone in a marriage-like relationship, and as employed if they reported working at least part-time. Smoking status was dichotomized according to whether a respondent reported being a current smoker (versus never smoked or former smoker). We used participants' responses to the following question as a dichotomous measure of general health status: "Do you have any medical problem(s) that interfered with your ability to exercise over the past twelve months?" Participants also reported whether they were currently taking medications for hypertension or cholesterol; in the case of cholesterol medications, we used detailed information on medication type to determine whether participants were taking statins, a class of medications that may affect C-reactive protein in addition to cholesterol.

Of particular interest for this analysis was a neighborhood environment questionnaire administered exclusively at the 2005-2006 exam. This questionnaire asked participants to report reasons for moving to their current residential neighborhood. Among the reasons from which respondents could select, three were related to the built environment: presence of amenities (e.g. parks, sidewalks), presence of stores and restaurants, and access to public transportation. We created a dichotomous variable to indicate whether a respondent selected one or more of these reasons for moving. This variable served as a proxy for neighborhood preferences and other residential choice factors that could confound the relationship between the built environment and health. 


\subsection{Statistical analyses}

All statistical analyses were conducted using Stata version 13.0. We examined descriptive statistics to describe sample characteristics, compare movers and non-movers, and assess characteristics of movers across levels of change in walkability resulting from residential relocation (i.e. decrease, minimal change, increase). We used the $\chi^{2}$ test, Fisher's exact test, or analysis of variance as appropriate to test for statistically significant differences.

We used fixed effects regression models (logistic for walking participation and atherogenic dyslipidemia, linear for all other outcomes) to estimate the associations between within-person change in walkability resulting from residential relocation and within-person change in each health outcome of interest. Fixed effects models rely exclusively on within-person variation over time, allowing each individual to serve as his or her own control. These models thus control for observed and unobserved time-invariant characteristics (Allison, 2005; Lovasi and Goldsmith, 2014). The fixed effects modeling strategy offers one way to control for unmeasured confounders, such as underlying preferences and attitudes that drive residential self-selection, to the extent that these types of unmeasured confounders remain stable within individuals over time.

The fixed effects models were adjusted only for time-varying covariates, as time-invariant characteristics are inherently controlled for (i.e. differenced out) due to the focus of these models on within-person variation. The general fixed effects equation for this analysis is presented in Eq. (3):

Health $_{i t}=\alpha_{i}+\beta$ Walkability $_{i t}+\delta$ Time $_{t}+\gamma Z_{i t}+\nu_{i t}$

where Health $h_{i t}$ is the health outcome of interest for individual $i$ at time $t$ (baseline or follow-up), $\alpha_{i}$ is an individual-specific intercept, Walkability $_{i t}$ is the time-varying walkability index, Time $_{t}$ represents the number of days between the baseline and follow-up exams, $Z_{i t}$ is a vector of time-varying covariates (income, household size, marital status, employment status, smoking status, health problems that interfere with physical activity), and $\nu_{i t}$ represents time-varying random error.

We compared our fixed effects models to random effects regression models, which account for correlated error terms within individuals observed at different time points; however, because they also incorporate differences between individuals, they differ from fixed effects models in that they do not control for unmeasured time-invariant characteristics (Cameron and Trivedi, 2005). Comparing the direction, magnitude, and statistical significance of the fixed effects and random effects coefficients allowed us to assess the presence and direction of bias from time-invariant unmeasured confounding.

The random effects models were adjusted for both time-varying and time-invariant characteristics, the latter of which are not removed through differencing as in fixed effects. The indicator of reasons for moving to the current neighborhood was considered to be timeinvariant because it was only available at the 2005-2006 exam, and thus could only be included in the random effects models. The general random effects equation is presented in Eq. (4):

Health $_{i t}=\alpha+\beta$ Walkability $_{i t}+$ STime $_{t}+\gamma Z_{i t}+\lambda C_{i}+\pi N_{i}+\mu_{i}+\nu_{i t}$

where Health ${ }_{i t}$ is the health outcome of interest for individual $i$ at time $t$ (baseline or follow-up), $\alpha$ is a common intercept for all individuals, Walkability ${ }_{i t}$ is the time-varying walkability index, Time $e_{t}$ represents the number of days between the baseline and follow-up exams, $Z_{i t}$ is a vector of time-varying covariates (income, household size, marital status, employment status, smoking status, health problems that interfere with physical activity), $C_{i}$ is a vector of time-invariant sociodemographic covariates (baseline age, gender, race/ ethnicity, highest educational attainment), $N_{i}$ is the binary indicator of reasons for moving to the current residential neighborhood, $\mu_{i}$ represents time-invariant individual-specific error, and $\nu_{i t}$ represents time-varying random error.

A key difference between the two types of models is the time-invariant error term $\mu_{i}$, which is differenced out of the fixed effects models but remains in the random effects models. If this term, which includes all unmeasured variables that remain constant within individuals over time, is correlated with the included independent variables, the random effects coefficients for those variables could be biased.

All regression models were estimated separately for each dependent variable. The standard errors for all models were clustered by geographic area to account for the fact that individuals were located in different areas of the U.S. Although all CARDIA participants were originally recruited in four cities, they have moved over time and were clustered in 135 Core Based Statistical Areas (CBSAs) at the 20052006 exam. We therefore specified the 2005-2006 CBSA as a cluster term in Stata in order to adjust our standard errors for potential intragroup correlation by metropolitan area.

In sensitivity analyses, we adjusted the systolic blood pressure model for self-reported use of hypertension medications, and the LDL cholesterol, HDL cholesterol, and C-reactive protein models for self-reported use of statins. We also tested the sensitivity of our results to the two alternative versions of the walkability index described in Section 2.2, which incorporated different weighting strategies for the index components.

We used the Hausman specification test (Hausman, 1978) to formally compare the fixed effects and random effects estimates. This test evaluates the null hypothesis that there is no correlation between unobserved individual-specific variation $\left(\mu_{i}\right)$ and the independent variables included in the model (i.e. the fixed effects and random effects estimates are statistically equal).

\section{Results}

\subsection{Summary statistics}

Walkability index values at participants' baseline (2000-2001) residential locations ranged from 2.02 to 68.51 with a mean of 20.14 (Table 1). Participants' new residential locations at follow-up (2005-2006) were slightly less walkable on average. Three-quarters of respondents reported participating in any walking activity at baseline, and walking exercise unit values ranged from 0 to 144 with a mean of 49.31. The proportion of participants engaging in any walking activity and the average value of walking exercise units were slightly 
Table 1

Descriptive statistics at baseline (2000-2001) and follow-up (2005-2006) exams, $n=1079$ middle-aged adults who moved residential locations between exams.

\begin{tabular}{|c|c|c|}
\hline Characteristics & $\begin{array}{l}\text { Baseline, mean } \\
\text { (SD) or \% }\end{array}$ & $\begin{array}{l}\text { Follow-up, mean } \\
\text { (SD) or \% }\end{array}$ \\
\hline \multicolumn{3}{|l|}{ Built environment exposure } \\
\hline Walkability index & $20.14(7.70)$ & $18.05(8.05)$ \\
\hline \multicolumn{3}{|l|}{ Health outcomes } \\
\hline \multicolumn{3}{|l|}{ Physical activity from walking } \\
\hline Participation (\%) & 75.72 & 71.08 \\
\hline Exercise units & $49.31(50.59)$ & $48.93(51.86)$ \\
\hline Body mass index, $\mathrm{kg} / \mathrm{m}^{2}$ & $28.41(6.50)$ & $29.19(6.69)$ \\
\hline Waist circumference, $\mathrm{cm}$ & $88.79(15.01)$ & $91.48(15.20)$ \\
\hline Systolic blood pressure, $\mathrm{mmHg}$ & $112.24(13.69)$ & $116.26(14.70)$ \\
\hline HOMA-IR (insulin resistance) $)^{\mathrm{a}}$ & $2.21(1.66)$ & $2.43(1.84)$ \\
\hline Triglycerides, mg/dL & $95.37(57.22)$ & $101.39(57.92)$ \\
\hline LDL cholesterol, mg/dL & $113.44(33.90)$ & $110.56(32.27)$ \\
\hline HDL cholesterol, mg/dL & $51.29(14.49)$ & $54.71(16.78)$ \\
\hline C-reactive protein, ug/mL & $3.11(5.06)$ & $2.74(5.15)$ \\
\hline Atherogenic dyslipidemiab $(\%)$ & 9.27 & 9.64 \\
\hline \multicolumn{3}{|l|}{ Sociodemographic covariates } \\
\hline Age, in years & $39.67(3.68)$ & $44.70(3.70)$ \\
\hline Female $(\%)$ & 54.77 & $-{ }^{c}$ \\
\hline White race/ethnicity (vs. black) (\%) & 49.12 & $-^{\mathrm{c}}$ \\
\hline More than high school (vs. less/equal) (\%) & 58.20 & $-{ }^{\mathrm{c}}$ \\
\hline Income, in thousands of U.S. dollars & $62.51(38.86)$ & $68.32(41.70)$ \\
\hline Household size & $3.04(1.61)$ & $2.86(1.51)$ \\
\hline Currently married (\%) & 46.80 & 48.66 \\
\hline Currently working (\%) & 85.82 & 80.44 \\
\hline \multicolumn{3}{|l|}{ Health covariates } \\
\hline Current smoker (\%) & 23.63 & 21.41 \\
\hline Health problems interfere with PA (\%) & 14.55 & 14.83 \\
\hline On medication(s) for hypertension (\%) & 6.58 & 14.94 \\
\hline On medication(s) for cholesterol (\%) & 1.11 & 7.41 \\
\hline \multicolumn{3}{|l|}{ Reasons for moving to neighborhood } \\
\hline Moved for built environment (\%) & $-{ }^{d}$ & 58.85 \\
\hline
\end{tabular}

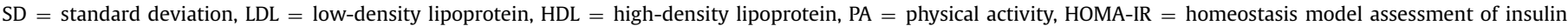
resistance.

a Participants with diabetes at baseline $(n=42)$ excluded from HOMA-IR calculation.

${ }^{\mathrm{b}}$ Atherogenic dyslipidemia is a classification of cardiovascular disease risk based on elevated triglycerides ( $>150 \mathrm{mg} / \mathrm{dL}$ ) and low $\mathrm{HDL}$ cholesterol ( $<50 \mathrm{mg} / \mathrm{dL}$ for women, $<40 \mathrm{mg} / \mathrm{dL}$ for men)

c These characteristics are considered to be time-invariant and thus have the same values for both exams.

d Reasons for moving to the current neighborhood were not measured at the 2000-2001 exam.

Table 2

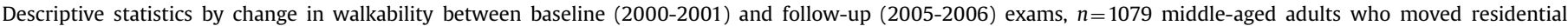
locations between exams.

\begin{tabular}{|c|c|c|c|c|c|}
\hline \multirow[t]{2}{*}{ Characteristics } & \multirow[t]{2}{*}{ All participants $(n=1079)$} & \multicolumn{4}{|c|}{ Groups by change in walkability index } \\
\hline & & Decrease $(n=394)^{\mathrm{a}}$ & Minimal change $(n=510)^{\mathrm{b}}$ & Increase $(n=175)^{c}$ & $\mathrm{p}$ \\
\hline \multicolumn{6}{|l|}{ Built environment variables } \\
\hline Walkability index at baseline & $20.14(7.70)$ & $23.24(7.14)$ & $19.14(6.95)$ & $16.07(8.37)$ & $0.00^{* * * * *}$ \\
\hline Change in walkability index & $-2.09(7.04)$ & $-8.99(4.87)$ & $-0.19(1.75)$ & $7.91(5.09)$ & - \\
\hline Moved for built environment (\%) & 58.85 & 54.06 & 62.35 & 59.43 & $0.04 * *$ \\
\hline \multicolumn{6}{|l|}{ Health outcomes at baseline } \\
\hline \multicolumn{6}{|l|}{ Physical activity from walking } \\
\hline Participation (\%) & 75.72 & 76.65 & 73.33 & 80.57 & 0.14 \\
\hline Exercise units & $49.31(50.59)$ & $49.26(50.48)$ & $49.44(51.88)$ & $49.05(47.18)$ & 0.99 \\
\hline Body mass index, $\mathrm{kg} / \mathrm{m}^{2}$ & $28.41(6.50)$ & $28.12(6.41)$ & $28.34(6.47)$ & $29.27(6.78)$ & 0.14 \\
\hline Waist circumference, $\mathrm{cm}$ & $88.79(15.01)$ & $88.44(14.63)$ & $88.51(15.40)$ & $90.43(14.68)$ & 0.29 \\
\hline Systolic blood pressure, $\mathrm{mmHg}$ & $112.24(13.69)$ & $111.00(12.81)$ & $112.92(14.22)$ & $113.07(13.78)$ & $0.08 *$ \\
\hline HOMA-IR (insulin resistance) d & $2.21(1.66)$ & $2.14(1.42)$ & $2.25(1.86)$ & $2.22(1.51)$ & 0.60 \\
\hline Triglycerides, mg/dL & $95.37(57.22)$ & $95.81(58.71)$ & $93.93(56.49)$ & $98.57(56.08)$ & 0.64 \\
\hline LDL cholesterol, mg/dL & $113.44(33.90)$ & $113.17(33.08)$ & $112.76(35.39)$ & $116.03(31.24)$ & 0.53 \\
\hline HDL cholesterol, mg/dL & $51.29(14.49)$ & $51.18(14.46)$ & $51.43(14.63)$ & $51.15(14.23)$ & 0.96 \\
\hline C-reactive protein, ug/mL & $3.11(5.06)$ & $2.77(3.91)$ & $3.35(6.00)$ & $3.16(4.27)$ & 0.23 \\
\hline Atherogenic dyslipidemia (\%) & 9.27 & 10.15 & 8.43 & 9.71 & 0.66 \\
\hline
\end{tabular}

lower at the follow-up exam. On average, participants at baseline were within the normal ranges for waist circumference, systolic blood pressure, HOMA-IR, triglycerides, and HDL cholesterol, but were overweight $(25 \leq \mathrm{BMI}<30)$ and slightly above optimal levels for LDL cholesterol (mean is 113.44 , optimal is $<100$ ) and C-reactive protein (mean is 3.11 , normal is $<3$ ). Approximately 7 percent of participants reported taking medication for hypertension and 1 percent reported taking medication for cholesterol at baseline. Nearly 60 percent of respondents reported choosing their new residential location for reasons related to the built environment. 


\begin{tabular}{|c|c|c|c|c|c|}
\hline \multirow[t]{2}{*}{ Characteristics } & \multirow{2}{*}{ All participants $(n=1079)$} & \multicolumn{4}{|c|}{ Groups by change in walkability index } \\
\hline & & Decrease $(n=394)^{a}$ & Minimal change $(n=510)^{\mathrm{b}}$ & Increase $(n=175)^{c}$ & $\mathrm{p}$ \\
\hline \multicolumn{6}{|l|}{ Change in health outcomes } \\
\hline Change in walking participation & & & & & 0.32 \\
\hline Started participating (\%) & 10.84 & 11.17 & 11.96 & 6.86 & \\
\hline Stopped participating (\%) & 15.48 & 16.75 & 14.90 & 14.29 & \\
\hline Change in walking exercise units & $-0.37(56.60)$ & $-3.72(55.38)$ & $2.07(57.96)$ & $0.02(55.20)$ & 0.31 \\
\hline Change in body mass index, $\mathrm{kg} / \mathrm{m}^{2}$ & $0.78(2.83)$ & $0.90(2.49)$ & $0.72(2.81)$ & $0.66(3.54)$ & 0.54 \\
\hline Change in waist circumference, $\mathrm{cm}$ & $2.68(8.28)$ & $2.91(8.49)$ & $2.50(8.14)$ & $2.71(8.22)$ & 0.76 \\
\hline Change in systolic blood pressure, $\mathrm{mmHg}$ & $4.02(13.07)$ & $4.95(12.47)$ & $3.77(14.00)$ & $2.62(11.37)$ & 0.12 \\
\hline Change in HOMA-IR ${ }^{d}$ & $0.23(1.57)$ & $0.32(1.51)$ & $0.18(1.67)$ & $0.13(1.40)$ & 0.31 \\
\hline Change in triglycerides, $\mathrm{mg} / \mathrm{dL}$ & $6.02(50.30)$ & $8.93(53.21)$ & $4.21(49.49)$ & $4.74(45.65)$ & 0.35 \\
\hline Change in LDL cholesterol, $\mathrm{mg} / \mathrm{dL}$ & $-2.88(26.82)$ & $-0.16(24.30)$ & $-6.13(29.09)$ & $0.44(24.23)$ & $0.00^{* * * * *}$ \\
\hline Change in HDL cholesterol, mg/dL & $3.42(10.20)$ & $3.18(10.02)$ & $3.73(10.59)$ & $3.08(9.43)$ & 0.64 \\
\hline Change in C-reactive protein, $\mathrm{ug} / \mathrm{mL}$ & $-0.37(5.09)$ & $-0.30(3.14)$ & $-0.73(5.16)$ & $0.52(7.70)$ & $0.02^{* * *}$ \\
\hline Change in atherogenic dyslipidemia & & & & & 0.58 \\
\hline New case $(\%)$ & 5.38 & 5.33 & 4.90 & 6.86 & \\
\hline Removed case (\%) & 5.00 & 6.09 & 4.12 & 5.14 & \\
\hline \multicolumn{6}{|l|}{ Covariates at baseline } \\
\hline Age, in years & $39.67(3.68)$ & $39.74(3.66)$ & $39.64(3.71)$ & $39.55(3.66)$ & 0.84 \\
\hline Female $(\%)$ & 54.77 & 53.81 & 55.69 & 54.29 & 0.85 \\
\hline Race/ethnicity & & & & & 0.41 \\
\hline Black, not Hispanic (\%) & 50.88 & 48.48 & 52.94 & 50.29 & \\
\hline White, not Hispanic (\%) & 49.12 & 51.52 & 47.06 & 49.71 & \\
\hline More than high school (vs. less/equal) (\%) & 58.20 & 58.12 & 59.02 & 56.00 & 0.78 \\
\hline Income, in thousands of U.S. dollars & $62.51(38.86)$ & $62.67(37.84)$ & $63.02(39.45)$ & $60.63(39.58)$ & 0.78 \\
\hline Household size & $3.04(1.61)$ & $2.96(1.47)$ & $3.15(1.71)$ & $2.89(1.60)$ & $0.08^{*}$ \\
\hline Currently married (\%) & 46.80 & 46.70 & 48.82 & 41.14 & 0.21 \\
\hline Currently working (\%) & 85.82 & 86.55 & 86.27 & 82.86 & 0.47 \\
\hline Current smoker (\%) & 23.63 & 23.86 & 23.73 & 22.86 & 0.97 \\
\hline Health problems (\%) & 14.55 & 12.94 & 15.10 & 16.57 & 0.47 \\
\hline On medication(s) for hypertension (\%) & 6.58 & 5.33 & 7.45 & 6.86 & 0.44 \\
\hline On medication(s) for cholesterol (\%) & 1.11 & 1.02 & 0.98 & 1.71 & 0.71 \\
\hline \multicolumn{6}{|l|}{ Change in covariates, as applicable } \\
\hline Change in income & $5.82(28.54)$ & $8.06(26.19)$ & $6.42(28.46)$ & $-0.99(32.66)$ & $0.00^{* * * * *}$ \\
\hline Change in household size & $-0.18(1.46)$ & $-0.10(1.35)$ & $-0.20(1.53)$ & $-0.28(1.50)$ & 0.33 \\
\hline Change in marital status & & & & & $0.01^{* *}$ \\
\hline New marriage $(\%)$ & 10.75 & 12.94 & 10.59 & 6.29 & \\
\hline No longer married (\%) & 8.90 & 6.85 & 8.63 & 14.29 & \\
\hline Change in employment status & & & & & 0.70 \\
\hline Started working (\%) & 6.12 & 6.09 & 5.49 & 8.00 & \\
\hline Stopped working (\%) & 11.49 & 12.44 & 11.37 & 9.71 & \\
\hline Change in smoking status & & & & & $0.06^{*}$ \\
\hline Started smoking (\%) & 2.59 & 1.52 & 2.35 & 5.71 & \\
\hline Stopped smoking (\%) & 4.82 & 4.82 & 5.49 & 2.86 & \\
\hline Change in health problems & & & & & 0.87 \\
\hline New problems (\%) & 9.55 & 10.41 & 8.82 & 9.71 & \\
\hline No longer problems (\%) & 9.27 & 8.38 & 9.61 & 10.29 & \\
\hline \multicolumn{6}{|l|}{ Change in medication use } \\
\hline Started hypertension meds. (\%) & 9.09 & 8.40 & 9.02 & 10.86 & 0.35 \\
\hline Started cholesterol meds. (\%) & 6.58 & 6.35 & 8.04 & 2.86 & 0.11 \\
\hline
\end{tabular}

$\mathrm{LDL}=$ low-density lipoprotein, HDL = high-density lipoprotein, HOMA-IR = homeostasis model assessment of insulin resistance

* Significant at $90 \%$ confidence.

*** Significant at $95 \%$ confidence

**** Significant at $99 \%$ confidence.

Groups are based on change in walkability, with cut points set at \pm 0.5 SD of the change variable $(+3.52$ and -3.52$)$.

a Decrease: change in walkability ranges from -35.95 to -3.53 .

${ }^{\mathrm{b}}$ Minimal change in walkability ranges from -3.50 to 3.51 .

${ }^{\mathrm{c}}$ Increase: change in walkability ranges from 3.58 to 40.88 .

${ }^{\mathrm{d}}$ Participants with diabetes at baseline $(n=42)$ excluded from HOMA-IR calculation.

The change in walkability accompanying residential relocation ranged from 36 points lower to 41 points higher, with the average participant moving to an area with 2.09 points lower walkability (Table 2). Approximately 11 percent of respondents started participating in walking physical activity during the time between exams, while approximately 15 percent stopped. While slight increases in BMI, waist circumference, systolic blood pressure, HOMA-IR, and triglycerides were observed, the time between exams was marked by average improvements in LDL and HDL cholesterol and C-reactive protein.

Descriptive statistics for selected baseline and change variables showed several statistically significant differences across levels of change in the walkability index (Table 2). Individuals experiencing an increase or minimal change in walkability were more likely to report moving to their current neighborhood for reasons related to the built environment compared to those experiencing a decrease in walkability. The only statistically significant group difference in baseline health was for systolic blood pressure, with those experiencing an increase in walkability 
Table 3

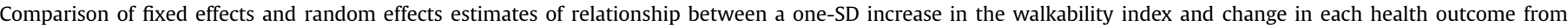
baseline to follow-up, $n=1079$ middle-aged adults who moved residential locations between 2000 and 2006 .

\begin{tabular}{|c|c|c|c|c|c|}
\hline \multirow[t]{2}{*}{ Outcome variables } & \multicolumn{2}{|l|}{ Fixed effects ${ }^{a}$} & \multicolumn{2}{|l|}{ Random effects ${ }^{b}$} & \multirow{2}{*}{$\begin{array}{l}\text { Hausman test } \\
\text { p-value }\end{array}$} \\
\hline & Coefficient $(S E)^{\mathrm{c}, \mathrm{d}}$ & p-value & Coefficient $(S E)^{\mathrm{c}, \mathrm{d}}$ & p-value & \\
\hline \multicolumn{6}{|l|}{ Walking physical activity } \\
\hline Participation & $-0.180(0.139)$ & 0.196 & $0.202(0.098)$ & $0.038^{* * *}$ & $0.003^{* * * * *}$ \\
\hline Exercise units & $-1.126(1.689)$ & 0.506 & $4.109(1.445)$ & $0.004^{* * * * *}$ & $0.021^{* * *}$ \\
\hline Body mass index, $\mathrm{kg} / \mathrm{m}^{2}$ & $-0.022(0.077)$ & 0.778 & $-0.018(0.069)$ & 0.793 & $0.000^{* * * * *}$ \\
\hline Waist circumference, $\mathrm{cm}$ & $-0.232(0.270)$ & 0.391 & $-0.260(0.242)$ & 0.283 & $0.000^{* * * * *}$ \\
\hline Systolic blood pressure, $\mathrm{mmHg}$ & $-0.810(0.373)$ & $0.032^{* *}$ & $-0.799(0.365)$ & $0.029^{* * *}$ & 0.294 \\
\hline $\ln (\text { HOMA-IR })^{f}$ & $-0.011(0.015)$ & 0.457 & $-0.016(0.010)$ & 0.104 & $0.005^{* * * * *}$ \\
\hline $\ln$ (triglycerides) & $-0.017(0.018)$ & 0.328 & $-0.025(0.015)$ & $0.084^{*}$ & $0.007^{* * * * *}$ \\
\hline LDL cholesterol, mg/dL & $-0.224(0.992)$ & 0.822 & $-1.506(0.790)$ & $0.057^{*}$ & 0.253 \\
\hline HDL cholesterol, mg/dL & $-0.121(0.331)$ & 0.715 & $0.256(0.284)$ & 0.367 & $0.009^{* * * * *}$ \\
\hline $\ln (C$-reactive protein) & $0.071(0.033)$ & $0.032^{* *}$ & $0.037(0.016)$ & $0.022^{* * *}$ & $0.057^{*}$ \\
\hline Atherogenic dyslipidemia & $0.343(0.231)$ & 0.137 & $-0.225(0.118)$ & $0.057^{*}$ & $0.037^{* * *}$ \\
\hline
\end{tabular}

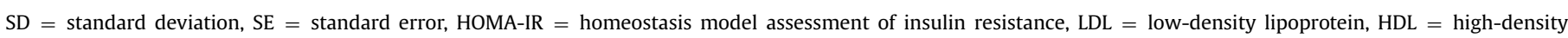
lipoprotein.

* Significant at $90 \%$ confidence.

** Significant at $95 \%$ confidence.

**** Significant at $99 \%$ confidence.

a Adjusted for time (days between exams) and time-varying sociodemographic and health covariates (income, household size, marital status, employment status, smoking status, health problems that interfere with physical activity)

${ }^{b}$ Adjusted for time (days between exams), sociodemographic and health covariates (baseline age, gender, race/ethnicity, educational attainment, income, household size,

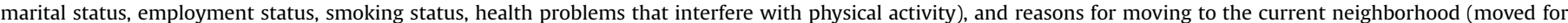
built environment reason(s))

c Standardized coefficients represent the association between a one-SD increase in the walkability index (i.e. increase of 7.95 units) and change in each health outcome in its specified units

d All standard errors clustered at the follow-up Combined Metropolitan Statistical Area (CMSA) level, with the exception of the fixed effects models for walking participation and atherogenic dyslipidemia (clustering option not available for fixed effects logistic models)

${ }^{\mathrm{e}}$ Hausman specification test evaluates null hypothesis that consistent (fixed effects) and efficient (random effects) estimates are equal; rejection favors fixed effects over random effects

${ }^{\mathrm{f}}$ Participants with diabetes at baseline $(n=42)$ excluded from HOMA-IR regressions

having the greatest average value. Between the baseline and follow-up exams, individuals experiencing an increase in walkability had the greatest increases in LDL cholesterol and C-reactive protein. Individuals experiencing an increase in walkability had smaller initial household sizes, had greater reductions in income, were more likely to have a marriage end, and were more likely to take up or resume smoking.

\subsection{Fixed effects and random effects regression results}

In the fixed effects models, increases in walkability from residential relocation were associated with decreases in systolic blood pressure and increases in C-reactive protein (Table 3). Specifically, a one-standard deviation increase in the walkability index (i.e. increase of 7.95 units) was associated with a $0.81 \mathrm{mmHg}$ decrease in systolic blood pressure and a 7.36 percent increase in C-reactive protein (coefficient from Table 3 exponentiated here due to log transformation of C-reactive protein data). No significant associations were observed between within-person change in walkability and within-person change in the remaining health outcomes included in this analysis.

In contrast, in the random effects models, walkability was positively associated with the odds of engaging in any walking physical activity, with walking exercise units, and with C-reactive protein; and inversely associated with systolic blood pressure, triglycerides, LDL cholesterol, and the odds of atherogenic dyslipidemia.

Hausman specification tests comparing the fixed effects and random effects estimates were statistically significant for the majority of models with the exceptions of systolic blood pressure and LDL cholesterol, suggesting the presence of unmeasured time-invariant confounding in the random effects regressions. The bias exerted by this confounding was away from the null in the waist circumference, insulin resistance (HOMA-IR), and triglycerides models; for these outcomes, the random effects coefficients were in the same direction but greater in absolute magnitude than the fixed effects estimates. The signs of the random effects and fixed effects estimates were in opposing directions for walking participation, walking exercise units, HDL cholesterol, and atherogenic dyslipidemia. However, the fixed effects estimates for these associations were statistically indistinguishable from zero, again suggesting bias away from the null in the random effects models. The random effects estimates were biased toward the null for BMI and C-reactive protein.

Sensitivity analyses adjusting for medication use in the systolic blood pressure, LDL cholesterol, HDL cholesterol, and C-reactive protein models produced results of similar magnitude, direction, and statistical significance (data not shown). The only noticeable difference in results was that the Hausman tests for systolic blood pressure and LDL cholesterol became statistically significant, indicating bias in the random effects models; this finding favored interpretation of the fixed effects coefficients, which were similar in direction and magnitude and showed the same patterns of statistical significance with and without adjustment for medication use. Sensitivity analyses testing alternative weighting strategies for the walkability index also produced similar results, although the coefficients for systolic blood pressure became somewhat smaller and, in the fixed effects models, statistically non-significant when all walkability components (i.e. density, street connectivity, and food/physical activity resources) were weighted equally in the composite index. 


\section{Discussion}

\subsection{Overview of associations}

We found neighborhood walkability to be moderately associated with certain aspects of cardiometabolic health in a sample of 1079 middle-aged adults who moved residential locations between 2000 and 2006 . When we estimated these associations using within-person changes in cardiometabolic health and objectively measured walkability (i.e. fixed effects), we found increases in walkability to be associated with reductions in systolic blood pressure and increases in C-reactive protein. The associations with systolic blood pressure were not consistently significant under alternative versions (i.e. weighting strategies) of the walkability index, although the selected index equation was specified based on theory and past research. While walkability was associated in the anticipated direction with several health outcomes in the random effects models, the results of the Hausman specification test indicated that these estimates were biased by unmeasured timeinvariant confounding for all outcomes other than systolic blood pressure and LDL cholesterol. The direction of this bias was most commonly away from the null or in the opposite direction of effect, suggesting that associations between walkability and health may be overstated or otherwise misrepresented in the absence of adequate controls for residential self-selection and other sources of unobserved heterogeneity.

The fixed effects coefficient for systolic blood pressure corresponds with a limited body of research that has observed associations between the built environment and blood pressure. This emerging evidence base includes two cross-sectional studies that found higher population and housing density (Chaix et al., 2008; Agyemang et al., 2007) and green space quality (Agyemang et al., 2007) to be correlated with lower systolic blood pressure, and a longitudinal study in which higher neighborhood walkability predicted decreases in systolic and diastolic blood pressure over a one-year follow-up period in middle-aged and older adults (Li et al., 2009).

The fixed effects coefficient for C-reactive protein, on the other hand, was in the unanticipated direction, suggesting that a move to a more walkable neighborhood was associated with increased (i.e. less healthy) C-reactive protein levels. This finding corresponds with the inverse (though statistically non-significant) associations observed between walkability and walking physical activity. This finding may also reflect the fact that C-reactive protein is adversely affected by stressors such as noise, sleep loss, overcrowding, and exposure to air pollution (King, 2013), measures of which were not available for this study. These stressors tend to be more prevalent in central urban areas, which also tend to have greater density, land use mix, and street connectivity. Thus, the positive association between walkability and C-reactive protein found in this study may be attributable in part to the detrimental aspects of dense urban environments. These results may also reflect the focus of this study on residential relocation, as moving tends to be a stressful life event that can be associated with a variety of negative health outcomes.

In a cross-sectional analysis, King (2013) found C-reactive protein to have a positive association with population density but an inverse association with land use mix, suggesting that different components of the walkable built environment "package" may entail competing health risks and benefits. As limited work to date has evaluated the relationship between the built environment and C-reactive protein, the present study adds a useful perspective to the evolving discourse on walkability and markers of inflammation.

\subsection{Comparison of fixed effects and random effects estimates: the role of unmeasured confounding}

The random effects coefficients for waist circumference, HOMA-IR, and triglycerides were larger in absolute magnitude than the fixed effects estimates for these outcomes, indicating bias away from the null; however, these differences were relatively small and none of these coefficient estimates were statistically significant at greater than 90 percent confidence. For walking participation, walking exercise units, HDL cholesterol, and atherogenic dyslipidemia, the signs of the random effects and fixed effects coefficients were in opposing directions; the fixed effects estimates for these outcomes were not statistically significant, suggesting again that the random effects estimates may be overstated.

One potential explanation for the biased random effects estimates in this study is residential self-selection: individuals who choose (or are constrained) to live in more walkable neighborhoods may do so for reasons related to walking and other health behaviors that could also affect the health outcomes considered in this analysis. We sought to account for residential self-selection in the random effects models by including an indicator of self-reported reasons for moving to a new residential neighborhood between the two CARDIA exams. Similar types of controls, generally focusing on neighborhood preferences, have been used in many past efforts to disentangle the influence of residential self-selection (Handy et al., 2008; Owen et al., 2007; Cerin et al., 2007; Frank et al., 2007). While this approach is attractive in its methodological simplicity, it presumes that individuals are able to act upon their built environment preferences when making residential location decisions, and may therefore oversimplify the complexities and competing priorities that characterize residential selection processes. Furthermore, it presumes that neighborhood preferences remain stable over time, and that individuals can correctly express their preferences and ignore cognitive dissonance and other motivations to modify their self-reported preferences (e.g., social desirability, expectation of rewards).

We compared random effects models that did and did not adjust for reasons for moving to the current neighborhood (Appendix Table A.2) in order to assess the impact of including this confounder in our analysis. In several models (walking participation, walking exercise units, waist circumference, LDL cholesterol, HDL cholesterol, C-reactive protein, and atherogenic dyslipidemia), the estimated coefficient on the walkability index was overstated when the indicator was not included as a covariate. This provides some indication of residential self-selection and suggests that the indicator of reasons for moving to the current neighborhood addressed a portion of the bias in the random effects models. However, the remaining bias in the random effects models, as evidenced by the Hausman specification test results, suggest that other sources of unmeasured confounding are relevant and that simple binary controls may be insufficient to account for neighborhood choice and other sources of individual heterogeneity.

The fixed effects models addressed residential self-selection by treating its unobserved determinants as time-invariant factors that could be differenced out of the estimating equation. However, it is important to recognize that the fixed effects estimates in this study remained vulnerable to bias from unmeasured time-varying confounding. This limitation may be particularly relevant to the neighborhood and behavioral preferences and constraints that can influence residential self-selection, as such factors may be subject to change over time and in response to new environmental settings. For instance, if individuals who move to more walkable neighborhoods develop positive perceptions of this type of built environment, their relevant attitudes and preferences should not be viewed as fixed over time. The potential for dynamic preferences is also a limitation in the random effects models, as reasons for moving to the current neighborhood were only measured at the 2005-2006 exam. 
The endogeneity of neighborhood selection and health is difficult to address, as random assignment of individuals to neighborhoods is not possible. However, estimates of the relationship between the built environment and health can be improved through careful research design and enhanced modeling techniques. While longitudinal data alone are not sufficient to address residential self-selection, longitudinal research designs that leverage natural experiments (e.g., temporary shocks related to construction or natural disasters) or approximate random assignment (e.g., randomized lotteries among applicants for new residential developments) offer potential ways to reduce the influence of self-selection. Additionally, sophisticated modeling techniques are available to examine the complex and potentially simultaneous pathways between attitudes and preferences, residential location, and health behaviors. For instance, structural equation modeling methods such as full information maximum likelihood (FIML), coupled with longitudinal data, may be particularly well suited to model the dynamic relationships that characterize residential self-selection and health (Boone-Heinonen et al., 2011).

\subsection{Focus on residential relocation}

Although the focus of this analysis on residential relocation created a useful longitudinal framework, it also posed several unique challenges for causal inference. As previously noted, moving is a substantial and potentially stressful life event that can affect health and behavior in a variety of ways. Furthermore, the focus on residential relocation creates an additional layer of self-selection, as individuals choose not only where to move, but also whether to move in the first place. The differences between movers and non-movers in the CARDIA cohort suggest that moving is correlated with lower socioeconomic status (potentially over-representing renters), which could produce biased estimates if these confounding influences are not fully captured in the set of observed covariates. The movers in this sample may also differ from non-movers on a variety of unobservable characteristics. Next, the results may not be generalizable to longitudinal changes in health among those who remain in the same neighborhood over time but nevertheless experience incremental changes to the built environment. Additionally, because data on time since moving were not available, the potential for moderation by length of time in the new residential location could not be tested.

\subsection{Divergence from previous work}

The results of our study contrast with those of Hirsch et al. (2014), who also used fixed effects models and found increases in Street Smart Walk Score ${ }^{\circledR}$ following residential relocation to be associated with increases in walking activity and reductions in BMI. These differences may be attributable to cohort life stage, as the previous study focused on older adults who may face different time commitments and health considerations than the middle-aged adults in our analysis. Hirsch et al. (2014) also examined transportation and leisure walking as separate outcomes, finding changes in walkability to be associated with the former but not the latter behavior. This distinction corresponds with past research that has found the built environment to have varying associations with utilitarian and recreational walking (Owen et al., 2007; Sundquist et al., 2011; Rodriguez et al., 2009; Christian et al., 2011). Given these distinct relationships, the lack of separate data on transportation and leisure walking for the CARDIA cohort is both a limitation and a potential explanation for divergence with past studies of walking behavior.

\subsection{Other limitations}

Several additional limitations relate to the methods used in this analysis. Fixed effects regression is less efficient when changes in exposures and outcomes over time are limited, which may be particularly relevant in an observational cohort setting with high tracking of health behaviors; limited changes in exposures and outcomes could lead to attenuated coefficient estimates. We also modeled each health outcome independently although the error terms for these equations could be correlated; correlations between outcomes are presented in Appendix Tables A.3-1 (baseline) and A.3-2 (follow-up). Next, as shown in Table 2, individuals moving to more walkable neighborhoods started from lower walkability index values, while those moving to less walkable neighborhoods started from higher index values. This suggests a tendency for individuals with very low or very high baseline walkability to move to neighborhoods of more moderate walkability, which could bias the coefficient estimates in this analysis. However, because we observed individuals at both end of this distribution, this bias may be limited. Additionally, the potential for chance associations should also be considered in light of the number of health outcomes examined in this analysis.

Furthermore, several types of data relevant to our research question were not available for the CARDIA cohort. First, we were not able to incorporate neighborhood-level air quality data due to the geographic extent of our sample, as existing secondary data sources do not readily capture neighborhood-level pollutants across the U.S. Next, while BMI and all cardiometabolic outcomes were measured objectively during inperson examinations, data on walking behavior were derived from self-report physical activity questionnaires that may be subject to recall and social desirability biases. However, participants generally reported a decrease in physical activity between the two exam years, corresponding with previous work in the CARDIA cohort that has found cardiorespiratory fitness to decrease over time (Carnethon et al., 2009). Finally, although past studies have suggested that perceived and objectively measured neighborhood attributes have distinct associations with travel behavior and physical activity (Giles-Corti et al., 2013; Sundquist et al., 2011; Leslie et al., 2005; Dill and Voros, 2007; Hoehner et al., 2005), data on environmental perceptions were only available for the 2005-2006 CARDIA exam and therefore could not be included in this longitudinal analysis.

\section{Conclusion}

We found increases in neighborhood walkability to be correlated with reductions in systolic blood pressure and increases in C-reactive protein among a sample of middle-aged adults in the U.S. who moved residential locations between 2000 and 2006 . The opposing directions of effect for systolic blood pressure and C-reactive protein illustrate the potential competing health risks (e.g., crowding, noise, air pollution, stress) and health benefits of dense, walkable environments. The differences between the random effects and fixed effects estimates in this analysis demonstrate that unmeasured time-invariant confounding, which could arise from residential self-selection, is an important threat to validity in this area of research. Approaches that base estimates on variation between individuals (e.g., random effects, cross-sectional research designs) may be subject to bias, which could overstate or otherwise misrepresent the relationship 
between walkability and health. Moreover, simple and static indicators of neighborhood choice and preferences may not fully account for the complex and dynamic determinants of residential self-selection; future survey efforts should therefore focus on developing and validating multidimensional, time-varying measures of built environment preferences. The results of this analysis indicate that findings from cross-sectional studies should be interpreted with caution, and that further research is needed to more fully understand the potential for built environment interventions to influence active transportation and corresponding health outcomes.

\section{Acknowledgments}

The authors have no financial or other conflicts of interest to disclose. L.M. Braun had full access to all of the data in the study and takes responsibility for the integrity of the data and the accuracy of data analysis. We are grateful to Mr. Marc Peterson of the Carolina Population Center Spatial Analysis Unit for the underlying Geographic Information Systems data. This work was funded by the National Heart, Lung, and Blood Institute (NHLBI) grants R01HL104580 and R01HL114091. The Coronary Artery Risk Development in Young Adults Study (CARDIA) is supported by contracts HHSN268201300025C, HHSN268201300026C, HHSN268201300027C, HHSN268201300028C, HHSN268201300029C, and HHSN268200900041C from the NHLBI, the Intramural Research Program of the National Institute on Aging (NIA), and an intra-agency agreement between NIA and NHLBI (AG0005). For general support, the authors are grateful to the Carolina Population Center, University of North Carolina at Chapel Hill (grant P2C HD050924 from the Eunice Kennedy Shriver National Institute of Child Health and Human Development [NICHD]), the Nutrition Obesity Research Center (NORC), University of North Carolina (grant P30DK56350 from the National Institute for Diabetes and Digestive and Kidney Diseases [NIDDK]), and the UNC Center for Environmental Health and Susceptibility (CEHS), University of North Carolina (grant P30ES010126 from the National Institute of Environmental Health Sciences [NIEHS]). The National Heart, Lung, and Blood Institute had input into design and conduct of the study; collection, management, analysis, and interpretation of the data; preparation of the manuscript, and decision to submit the manuscript for publication.

\section{Appendix A}

See Tables A1, A2 and Tables A3-1, A3-2.

Table A1

Comparison of included cases (movers with complete data) and excluded cases (non-movers, movers with incomplete data) on baseline characteristics.

\begin{tabular}{|c|c|c|c|}
\hline \multirow[t]{2}{*}{ Characteristics } & \multirow{2}{*}{$\begin{array}{l}\text { Included cases } \\
\text { Movers with complete data }(n=1079)\end{array}$} & \multicolumn{2}{|l|}{ Excluded cases $^{\mathrm{a}}$} \\
\hline & & Non-movers $(n=1661)$ & Movers with incomplete data $(n=429)$ \\
\hline \multicolumn{4}{|l|}{ Built environment exposure } \\
\hline Walkability index & $20.14(7.70)$ & $19.73(7.53)$ & $20.26(7.90)$ \\
\hline \multicolumn{4}{|l|}{ Health outcomes } \\
\hline \multicolumn{4}{|l|}{ Physical activity from walking } \\
\hline Participation (\%) & 75.72 & 76.27 & 76.47 \\
\hline Exercise units & $49.31(50.59)$ & $49.61(51.10)$ & $50.54(51.86)$ \\
\hline Body mass index, $\mathrm{kg} / \mathrm{m}^{2}$ & $28.41(6.50)$ & $28.77(6.81)$ & $29.10(7.39)^{*}$ \\
\hline Waist circumference, $\mathrm{cm}$ & $88.79(15.01)$ & $89.09(14.91)$ & $90.63(16.59)^{* * *}$ \\
\hline Systolic blood pressure, $\mathrm{mmHg}$ & $112.24(13.69)$ & $112.94(15.18)$ & $114.20(15.26)^{* * *}$ \\
\hline HOMA-IR (insulin resistance) & $2.30(1.81)$ & $2.41(2.14)$ & $2.94(3.49)^{* * * *}$ \\
\hline Triglycerides, mg/dL & $95.37(57.22)$ & $104.63(96.72)^{* * * * *}$ & $126.60(139.34)^{* * * * *}$ \\
\hline LDL cholesterol, mg/dL & $113.44(33.90)$ & $112.88(30.71)$ & $114.13(34.80)$ \\
\hline HDL cholesterol, mg/dL & $51.29(14.49)$ & $50.83(14.51)$ & $49.54(15.03)^{* * *}$ \\
\hline C-reactive protein, ug/mL & $3.11(5.06)$ & $3.29(5.87)$ & $3.33(5.01)$ \\
\hline Atherogenic dyslipidemia (\%) & 9.27 & $11.56^{*}$ & $14.45^{\text {***** }}$ \\
\hline \multicolumn{4}{|l|}{ Sociodemographic covariates } \\
\hline Age, in years & $39.67(3.68)$ & $40.76(3.45)^{* * * * * *}$ & $39.86(3.69)$ \\
\hline Female $(\%)$ & 54.77 & $58.34 *$ & 55.94 \\
\hline White race/ethnicity (vs. black) (\%) & 49.12 & $60.14^{* * * * *}$ & 51.52 \\
\hline More than high school (vs. less/equal) (\%) & 58.20 & $65.02^{* * * *}$ & 55.40 \\
\hline Income, in thousands of U.S. dollars & $62.51(38.86)$ & $73.29(37.77)^{* * * * *}$ & $57.94(37.71)^{* * *}$ \\
\hline Household size & $3.04(1.61)$ & $3.26(1.47)^{* * * k \cdots}$ & $3.03(1.60)$ \\
\hline Currently married (\%) & 46.80 & 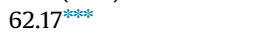 & 46.59 \\
\hline Currently working (\%) & 85.82 & 87.12 & $82.05^{*}$ \\
\hline \multicolumn{4}{|l|}{ Health covariates } \\
\hline Current smoker (\%) & 23.63 & 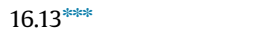 & 26.81 \\
\hline Health problems interfere with PA (\%) & 14.55 & $16.93^{*}$ & 16.86 \\
\hline On medication(s) for hypertension (\%) & 6.58 & 7.71 & 7.71 \\
\hline On medication(s) for cholesterol (\%) & 1.11 & $2.65^{* *}$ & $2.56^{* * *}$ \\
\hline
\end{tabular}

LDL = low-density lipoprotein, HDL = high-density lipoprotein, PA = physical activity, HOMA-IR = homeostasis model assessment of insulin resistance.

* Significant at $90 \%$ confidence.

** Significant at $95 \%$ confidence.

**** Significant at $99 \%$ confidence.

a Significance levels for group differences (relative to included cases) based on p-value from ANOVA for continuous variables, $\chi^{2}$ test for categorical variables. 
Table A2

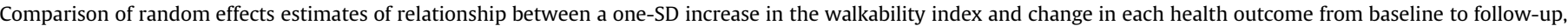
$n=1079$ middle-aged adults who moved residential locations between 2000 and 2006 .

\begin{tabular}{|c|c|c|c|c|}
\hline \multirow[t]{2}{*}{ Outcome variables } & \multicolumn{2}{|c|}{ Without reasons for moving to current neighborhood ${ }^{a}$} & \multicolumn{2}{|c|}{ With reasons for moving to current neighborhood ${ }^{\mathrm{b}}$} \\
\hline & Coefficient $(S E)^{c, d}$ & p-value & Coefficient $(\mathrm{SE})^{\mathrm{c}, \mathrm{d}}$ & p-value \\
\hline \multicolumn{5}{|l|}{ Walking physical activity } \\
\hline Participation & $0.229(0.102)$ & $0.024^{* * *}$ & $0.202(0.098)$ & $0.038^{* * *}$ \\
\hline Exercise units & $4.718(1.457)$ & $0.001^{* * * * * *}$ & $4.109(1.445)$ & $0.004^{* * * * *}$ \\
\hline Body mass index, $\mathrm{kg} / \mathrm{m}^{2}$ & $-0.017(0.069)$ & 0.805 & $-0.018(0.069)$ & 0.793 \\
\hline Waist circumference, $\mathrm{cm}$ & $-0.282(0.248)$ & 0.254 & $-0.260(0.242)$ & 0.283 \\
\hline Systolic blood pressure, $\mathrm{mmHg}$ & $-0.731(0.368)$ & $0.047^{* *}$ & $-0.799(0.365)$ & $0.029^{* *}$ \\
\hline $\ln (\text { HOMA-IR })^{\mathrm{e}}$ & $-0.016(0.010)$ & 0.116 & $-0.016(0.010)$ & 0.104 \\
\hline $\ln$ (triglycerides) & $-0.024(0.015)$ & 0.113 & $-0.025(0.015)$ & $0.084^{*}$ \\
\hline LDL cholesterol, mg/dL & $-1.531(0.812)$ & $0.059^{*}$ & $-1.506(0.790)$ & $0.057^{*}$ \\
\hline HDL cholesterol, mg/dL & $0.271(0.309)$ & 0.381 & $0.256(0.284)$ & 0.367 \\
\hline $\ln (C$-reactive protein $)$ & $0.040(0.016)$ & $0.009^{* * * * *}$ & $0.037(0.016)$ & $0.022^{* *}$ \\
\hline Atherogenic dyslipidemia & $-0.268(0.127)$ & $0.035^{* * * *}$ & $-0.225(0.118)$ & $0.057^{*}$ \\
\hline
\end{tabular}

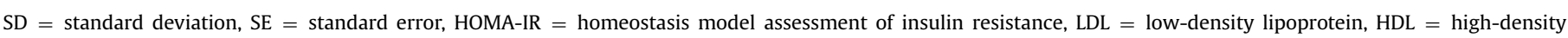
lipoprotein.

* Significant at $90 \%$ confidence.

** Significant at $95 \%$ confidence.

**** Significant at $99 \%$ confidence.

a Adjusted for time (days between exams), sociodemographic and health covariates (baseline age, gender, race/ethnicity, educational attainment, income, household size, marital status, employment status, smoking status, and health problems that interfere with physical activity).

${ }^{\mathrm{b}}$ Adjusted for the above covariates as well as reasons for moving to the current neighborhood (moved for built environment reason(s)).

c Standardized coefficients represent the association between a one-SD increase in the walkability index (i.e. increase of 7.95 units) and change in each health outcome in its specified units.

${ }^{\mathrm{d}}$ All standard errors clustered at the follow-up Core Based Statistical Area (CBSA) level.

e Participants with diabetes at baseline $(n=42)$ excluded from HOMA-IR regressions.

Table A.3-1

Correlation between health outcomes at baseline (2000-2001) exam, $n=1079$ middle-aged adults who moved residential locations between 2000 and 2006 .

\begin{tabular}{|c|c|c|c|c|c|c|c|c|c|c|c|}
\hline & Walk. partic. & Walk. EUs & BMI & Waist circum. & Syst. BP & HOMA-IR & Trig. & LDL & HDL & C-RP & Ath. dyslip. \\
\hline Walking participation & 1.000 & & & & & & & & & & \\
\hline Walking exercise units & 0.552 & 1.000 & & & & & & & & & \\
\hline Body mass index & -0.056 & -0.067 & 1.000 & & & & & & & & \\
\hline Waist circumference & -0.079 & -0.084 & 0.083 & 1.000 & & & & & & & \\
\hline Systolic blood pressure & -0.051 & -0.030 & 0.315 & 0.351 & 1.000 & & & & & & \\
\hline HOMA-IR & -0.047 & -0.081 & 0.552 & 0.553 & 0.241 & 1.000 & & & & & \\
\hline Triglycerides & 0.000 & 0.003 & 0.227 & 0.347 & 0.176 & 0.302 & 1.000 & & & & \\
\hline LDL cholesterol & -0.020 & -0.035 & 0.175 & 0.228 & 0.103 & 0.099 & 0.204 & 1.000 & & & \\
\hline HDL cholesterol & 0.025 & 0.015 & -0.291 & -0.414 & -0.090 & -0.318 & -0.412 & -0.151 & 1.000 & & \\
\hline C-reactive protein & -0.008 & -0.027 & 0.457 & 0.358 & 0.168 & 0.314 & 0.081 & 0.066 & -0.140 & 1.000 & \\
\hline Atherogenic dyslipidemia & -0.043 & -0.031 & 0.165 & 0.242 & 0.076 & 0.216 & 0.682 & 0.067 & -0.356 & 0.027 & 1.000 \\
\hline
\end{tabular}

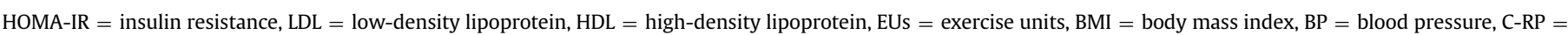
C-reactive protein.

Table A.3-2

Correlation between health outcomes at follow-up (2005-2006) exam, $n=1079$ middle-aged adults who moved residential locations between 2000 and 2006 .

\begin{tabular}{|c|c|c|c|c|c|c|c|c|c|c|c|}
\hline & Walk. partic. & Walk. EUs & BMI & Waist circum. & Syst. BP & HOMA-IR & Trig. & LDL & HDL & C-RP & Ath. dyslip. \\
\hline Walking participation & 1.000 & & & & & & & & & & \\
\hline Walking exercise units & 0.602 & 1.000 & & & & & & & & & \\
\hline Body mass index & -0.066 & -0.104 & 1.000 & & & & & & & & \\
\hline Waist circumference & -0.085 & -0.103 & 0.860 & 1.000 & & & & & & & \\
\hline Systolic blood pressure & -0.072 & -0.075 & 0.197 & 0.265 & 1.000 & & & & & & \\
\hline HOMA-IR & -0.095 & -0.101 & 0.430 & 0.478 & 0.100 & 1.000 & & & & & \\
\hline Triglycerides & -0.038 & -0.022 & 0.205 & 0.349 & 0.152 & 0.318 & 1.000 & & & & \\
\hline LDL cholesterol & -0.024 & -0.078 & 0.123 & 0.156 & 0.041 & 0.046 & 0.185 & 1.000 & & & \\
\hline HDL cholesterol & 0.091 & 0.072 & -0.314 & -0.440 & -0.092 & -0.294 & -0.429 & -0.174 & 1.000 & & \\
\hline C-reactive protein & -0.006 & -0.044 & 0.354 & 0.300 & 0.044 & 0.192 & 0.026 & -0.005 & -0.148 & 1.000 & \\
\hline Atherogenic dyslipidemia & -0.013 & -0.014 & 0.138 & 0.223 & 0.027 & 0.276 & 0.644 & 0.046 & -0.382 & 0.008 & 1.000 \\
\hline
\end{tabular}

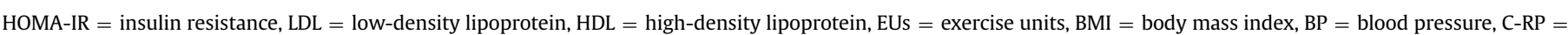
C-reactive protein. 


\section{References}

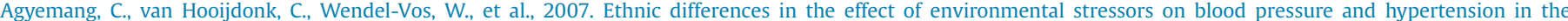
Netherlands. BMC Public Health 7, 118.

Allison, P.D., 2005. Fixed Effects Regression Methods for Longitudinal Data Using SAS. SAS Press, Cary, NC.

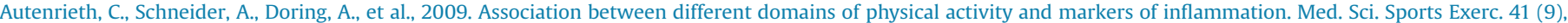
1706-1713.

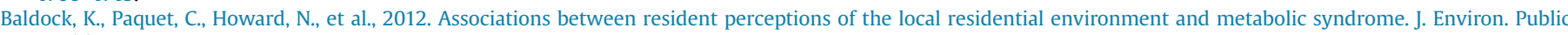
Health, 589409.

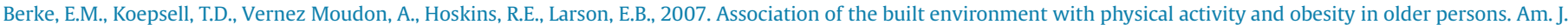
Public Health 97, 486-492.

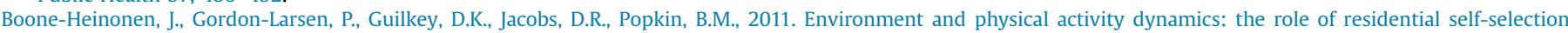
Psychol. Sport Exerc. 12, 54-60.

Cameron, A.C., Trivedi, P.K., 2005. Microeconomics: Methods and Applications. Cambridge University Press, New York.

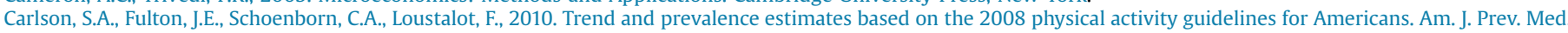
39 (4), 305-313.

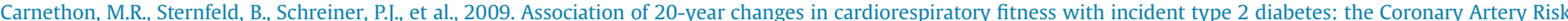
Development in Young Adults (CARDIA) fitness study. Diabetes Care 32, 1284-1288.

Carr, L.J., Dunsiger, S.I., Marcus, B.H., 2011. Validation of Walk Score for estimating access to walkable amenities. Br. J. Sports Med. 45, 1144-1148.

Carr, L.J., Dunsiger, S.I., Marcus, B.H., 2010. Walk Score ${ }^{\mathrm{TM}}$ as a global estimate of neighborhood walkability. Am. J Prev. Med. 39 (5), $460-463$.

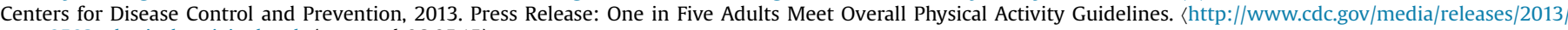
p0502-physical-activity.html (accessed 06.05.15).

Cerin, E., Leslie, E., du Toit, L., Owen, N., Frank, L.D., 2007. Destinations that matter: associations with walking for transport. Health Place 13 (3), $713-724$.

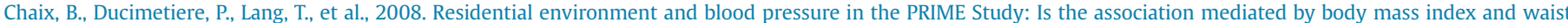
circumference? J. Hypertens. 26, 1078-1084.

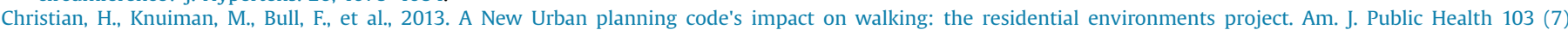
$1219-1228$.

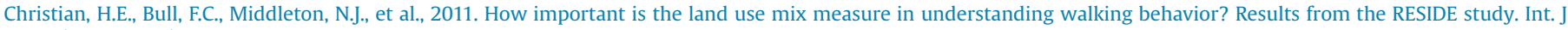
Behav. Nutr. Phys. Act. 8, 55.

Coffee, N.T., Howard, N., Paquet, C., Hugo, G., Daniel, M., 2013. Is walkability associated with a lower cardiometabolic risk? Health Place 21, $163-169$.

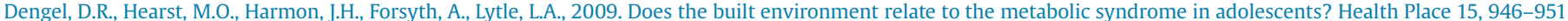

Dill, J., Voros, K., 2007. Factors affecting bicycling demand: Initial survey findings from the Portland, Oregon, region. Transp. Res. Rec. 2031 (1), 9-17.

Dun \& Bradstreet, Inc., 2013. Dun's Market Identifiers, Short Hills, NJ, United States.

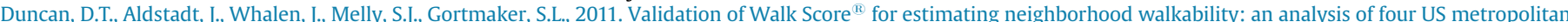
areas. Int. J. Environ. Res. Public Health 8, 4160-4179.

Ewing, R., Cervero, R., 2010. Travel and the built environment. J Am. Plan. Assoc. 76 (3), 265-294.

Frank, L.D., Andresen, M.A., Schmid, T.L., 2004. Obesity relationships with community design, physical activity, and time spent in cars. Am. J. Prev. Med. 27 (2), 87-96.

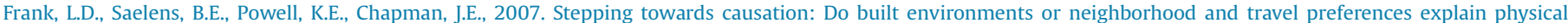
activity, driving, and obesity? Soc. Sci. Med. 65, 1898-1914.

Front Seat Management, 2014. LLC. Street Smart Walk Score. 〈http://www.walkscore.com/professional/street-smart.php〉.

Garden, F.L., Jalaludin, B.B., 2008. Impact of urban sprawl on overweight, obesity, and physical activity in Sydney, Australia. J. Urban Health 86 (1), 19-30.

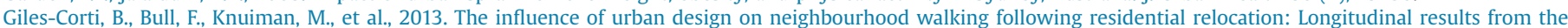
RESIDE study. Soc. Sci. Med. 77, 20-30.

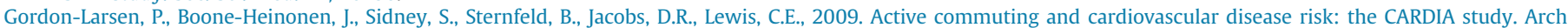
Intern. Med. 169 (13), 1216-1223.

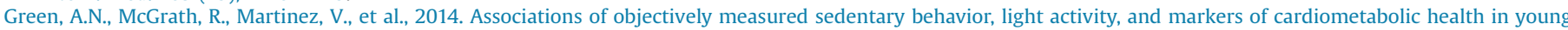
women. Eur. J. Appl. Physiol. 114 (5), 907-919.

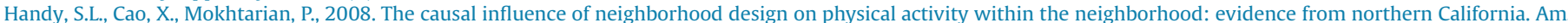
J. Health Promot. 22 (5), 350-358.

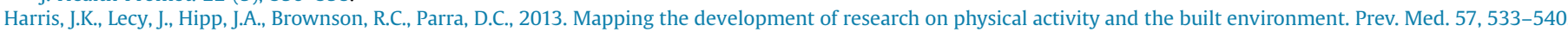

Hausman, J.A., 1978. Specification tests in econometrics. Econometrica 46, 1251-1271.

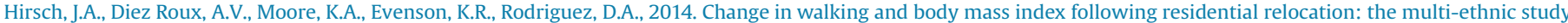
of atherosclerosis. Am. J Public Health 104 (3), e49-e56.

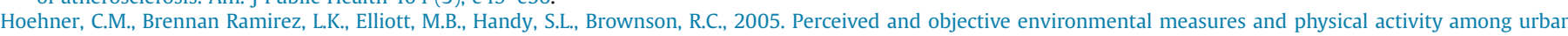
adults. Am. J. Prev. Med 28 (2), 105-116.

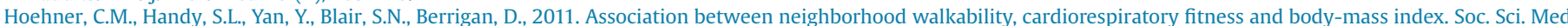
73, 1707-1716.

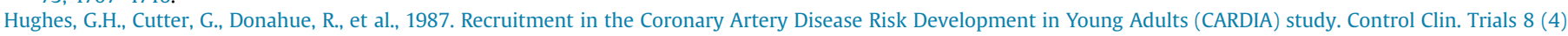
$68 \mathrm{~S}-73 \mathrm{~S}$.

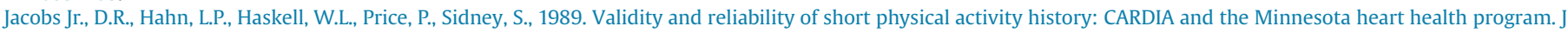
Cardiopulm. Rehabil. 9 (11), 448-459.

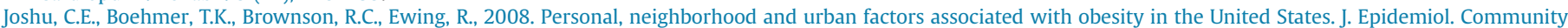
Health 62, 202-208.

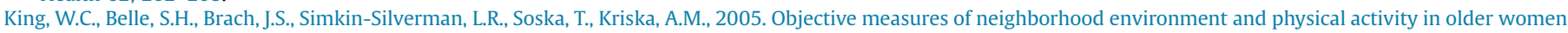
Am. J. Prev. Med. 28 (5), 461-469.

King, K., 2013. Neighborhood walkable urban form and C-reactive protein. Prev. Med. 57 (6), 850-854.

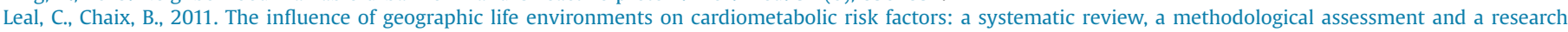
agenda. Obes. Rev. 12, 217-230.

Lee, I., Ewing, R., Sesso, H.D., 2009. The built environment and physical activity levels: the Harvard alumni health study. Am. J. Prev. Med. 37 (4), 293-298.

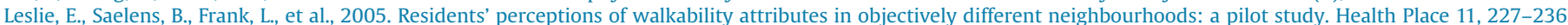

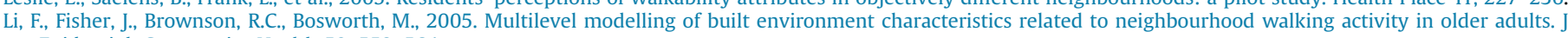
Epidemiol. Community Health 59, 558-564.

Li, F., Harmer, P., Cardinal, B.J., Vongjaturapat, N., 2009. Built environment and changes in blood pressure in middle aged and older adults, Prev. Med. 48, 237-241.

Li, F., Harmer, P.A., Cardinal, B.J., et al., 2008. Built environment, adiposity, and physical activity in adults aged 50-75. Am. J. Prev. Med. 35 (1), $38-46$.

Lovasi, G.S., Goldsmith, J., 2014. Invited commentary: Taking advantage of time-varying neighborhood environments. Am. J. Epidemiol. 180 (5), $462-466$.

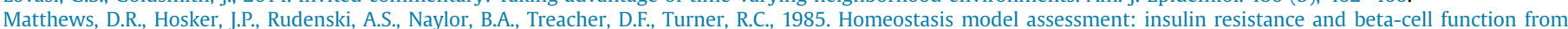
fasting plasma glucose and insulin concentrations in man. Diabetologia 28 (7), 412-419.

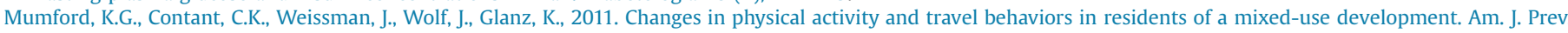
Med. 41 (5), 504-507.

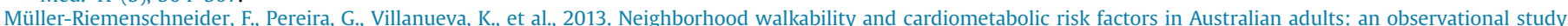
BMC Public Health 13, 755.

Owen, N., Cerin, E., Leslie, E., et al., 2007. Neighborhood walkability and the walking behavior of Australian adults. Am. J. Prev. Med. 33, 387-395.

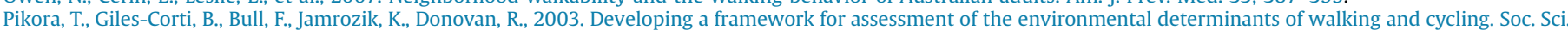
Med. 56 (8), 1693-1703. 


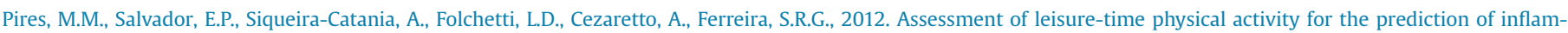
matory status and cardiometabolic profile. J. Sci. Med. Sport 15, 511-518.

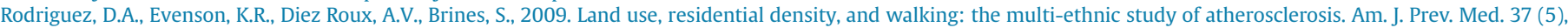
397-404.

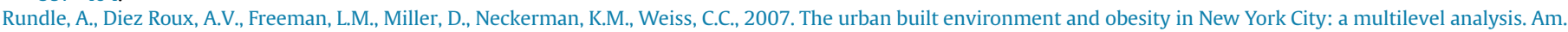
J. Health Promot. 21, 326-334.

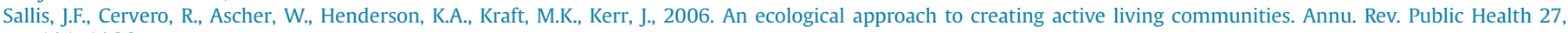
14.1-14.26.

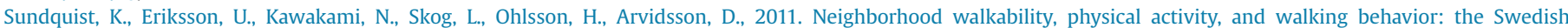
Neighborhood and Physical Activity (SNAP) study. Soc. Sci. Med. 72, 1266-1273.

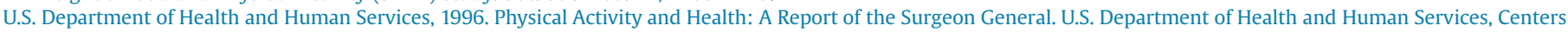
for Disease Control and Prevention, National Center for Chronic Disease Prevention and Health Promotion, Atlanta, GA.

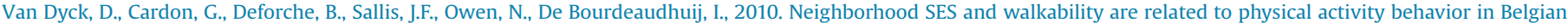
adults. Prev. Med. 50 (Suppl. 1), S74-S79.

Wells, N.M., Yang, Y., 2008. Neighborhood design and walking: a quasi-experimental longitudinal study. Am. J Prev. Med. 34 (4), $313-319$.

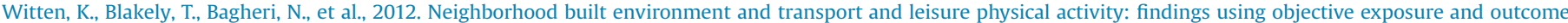
measures in New Zealand. Environ. Health Perspect. 120 (7), 971-977. 